\title{
Hepatic NAD salvage pathway is enhanced in mice on a high-fat diet
}

\author{
Melanie Penke ${ }^{\mathrm{a}, \mathrm{b}, *}$, Per S. Larsen ${ }^{\mathrm{c}}$, Susanne Schuster ${ }^{\mathrm{a}, \mathrm{b}}$, Morten Dall ${ }^{\mathrm{c}}$, Benjamin \\ A.H. Jensen ${ }^{\mathrm{d}}$, Theresa Gorski ${ }^{\mathrm{a}}$, Andrej Meusel ${ }^{\mathrm{d}, \mathrm{e}}$, Sandy Richter ${ }^{\mathrm{a}}$, \\ Sara G. Vienberg ${ }^{\text {c }}$, Jonas T. Treebak ${ }^{\mathrm{c}}$, Wieland Kiess ${ }^{\mathrm{a}, \mathrm{b}}$, Antje Garten ${ }^{\mathrm{a}}$ \\ a Center for Pediatric Research Leipzig (CPL), University Hospital for Children \& Adolescents, University of Leipzig, Liebigstr. 21, 04103 Leipzig, Germany \\ ${ }^{\mathrm{b}}$ LIFE Leipzig Research Centre for Civilization Diseases, University of Leipzig, Philipp-Rosenthalstr. 27, D-04103 Leipzig, Germany \\ ${ }^{\mathrm{c}}$ The Novo Nordisk Foundation Center for Basic Metabolic Research, Section of Integrative Physiology, Faculty of Health and Medical Sciences, University of \\ Copenhagen, Copenhagen, Denmark \\ d Department of Biology, Laboratory for Genomics and Molecular Biomedicine, Faculty of Science, University of Copenhagen, Copenhagen, Denmark \\ ${ }^{\mathrm{e}}$ Institut für Medizinische Physik und Biophysik, University of Leipzig, Härtelstr. 16-18, 04107 Leipzig, Germany
}

abstract

\begin{abstract}
* Corresponding author. Center for Pediatric Research Leipzig (CPL) University Hospital for Children \& Adolescents, University of Leipzig, Liebigstraße 21, 04103 Leipzig, Germany. Tel: $+49 / 3419726$ Tel.: +49/341 97 26513; fax: $+49 / 34197$

E-mail address

melanie.penke@medizin.uni-leipzig.de
\end{abstract} (M. Penke).

\begin{abstract}
Nicotinamide phosphoribosyltransferase (Nampt) is the rate-limiting enzyme for NAD salvage and the abundance of Nampt has been shown to be altered in non-alcoholic fatty liver disease. It is, however, unknown how hepatic Nampt is regulated in response to accumulation of lipids in the liver of mice fed a high-fat diet (HFD). HFD mice gained more weight, stored more hepatic lipids and had an impaired glucose tolerance compared with control mice. NAD levels as well as Nampt mRNA expression, protein abundance and activity were significantly increased in HFD mice. Enhanced NAD levels were associated with deacetylation of p53 and Nfkb indicating increased activation of Sirt1. Despite impaired glucose tol- erance and increased hepatic lipid levels in HFD mice, NAD metabolism was significantly enhanced. Thus, improved NAD metabolism may be a compensatory mechanism to protect against negative impact of hepatic lipid accumulation.
\end{abstract}

\section{Introduction}

More than $30 \%$ of Western adult population is affected by nonalcoholic fatty liver diseases (NAFLD) with a rapidly increasing number in obese and diabetic people. NAFLD covers a wide range of conditions from simple steatosis caused by accumulation of tri- glycerides to non-alcoholic steatohepatitis (NASH) which is associated with inflammatory processes. NASH is a risk factor for fibrosis and cirrhosis which are associated with a significant liver-related mor- tality and a median survival of 6 years (Tarantino and Finelli, 2013). The pathological pathways leading to NASH are not yet fully un- derstood. Interestingly, specific sirtuins (Sirts) seem to affect the development of NAFLD by regulating processes like hepatic gluco- neogenesis, mitochondrial biogenesis and fatty acid synthesis. Liver- specific Sirt1 knockout mice develop hepatic steatosis and show impaired insulin signaling on a high-fat diet (HFD) (Purushotham et al., 2009), while global transgenic mice overexpressing Sirt1 are pro- tected against the negative effects of a HFD such as inflammation, impaired glucose tolerance and hepatic steatosis (Pfluger et al., 2008).

Additionally, whole-body Sirt3 knockout mice show enhanced storage of hepatic triglycerides and insulin resistance (Hirschey et al., 2010) although liver-specific Sirt3 knockout mice do not display an overt phenotype (Fernandez-Marcos et al., 2012).

Sirtuins are nicotinamide adenine dinucleotide (NAD) depen- dent in respect to their deacetylase activity. The key enzyme in the mammalian NAD salvage pathway starting from nicotinamide is nico- tinamide phosphoribosyltransferase (Nampt) which converts nicotinamide to nicotinamide mononucleotide (NMN), an interme- diate in NAD biosynthesis (Revollo et al., 2007a). Additionally, Nampt is secreted from adipocytes (Tanaka et al., 2007), hepatocytes (Garten et al., 2010), and leucocytes (Friebe et al., 2011). Nampt circulates in the blood where it acts as a NMN biosynthetic enzyme (Revollo et al., 2007b) and/or cytokine (Samal et al., 1994). Several human studies have investigated how Nampt protein levels in biopsies and blood are affected in individuals with steatosis and $\mathrm{NASH}$, however, results are conflicting (Auguet et al., 2013; Dahl et al., 2010; Kukla et al., 2010).

It has been shown that rats fed a HFD develop fatty liver and hepatic insulin resistance already after 3 days of HFD feeding (Samuel et al., 2004). Moreover, another study in mice showed that hepatic insulin resistance is present already after 3 days on a HFD before insulin re- sistance develops in muscle and adipose tissue (Turner et al., 2013). Thus, hepatic insulin resistance develops very rapidly, but whether NAD metabolism is also affected in the early stages of the development of NAFLD is unknown. We hypothesize a link between the develop- ment of NAFLD and the ability to maintain NAD levels. To define the effect of lipid accumulation on hepatic NAD metabolism, we fed mice a HFD for 11 weeks and examined the effect on hepatic NAD me- tabolism. Despite hepaticlipid accumulation and impaired whole body glucose tolerance in mice fed a HFD, we found an improved hepatic NAD metabolism suggesting that this may be a compensatory mech- anism to protect against the negative impact of hepatic lipid accumulation on inflammation and apoptosis.

\section{Methods and procedures}

\subsection{Mice and Ethical approval}

All experiments were approved by the Danish Animal Experi- mental Inspectorate and complied with the EU convention for protection of vertebra animals used for scientific purposes (Council of Europe 123, Strasbourg, France, 1985). Twenty-four male mice were obtained from Taconic when they were 10 weeks of age (C57BL/ 6Ntac, \#DIO-B6). At the time of arrival in the laboratory, mice had been on a HFD (Research Diets Industry, D12492) or corresponding chow diet (Ctl) for 4 weeks. Mice were kept in a 12:12-h light:dark cycle and kept on the diet for additional 7 weeks. Water was given adlibitum. Mice were either single-(s) or group-housed (gr). To confirm that housing conditions (i.e., single- vs. group-housing) did not affect NAD metabolism, we measured Nampt protein abundance and NAD levels in a separate cohort of mice. In this cohort we applied 26 care- fully weight matched male mice (C57BL/6JBomTac) at 7 weeks of age. Upon arrival, the mice were acclimatized on a standard chow diet for 10 days before they were randomly assigned to the experimental groups and fed either a HFD (D12492) or a corresponding low fat diet (D12450J) for 11 weeks. The mice were either single-housed ( 5 mice per group per diet) or group-housed ( 8 mice per group per diet, 4 mice per cage) from the point of arrival to the day of termination. Housing conditions did not affect hepatic Nampt protein and NAD levels (Fig. $\mathrm{S} 1 \mathrm{~A}, \mathrm{~B})$. After 11 weeks on their respective diets, mice were anesthetized (Pentobarbital, $100 \mathrm{mg} / \mathrm{kg}$ body weight) and livers were carefully dissected, frozen in liquid nitrogen, and stored at $-80^{\circ} \mathrm{C}$ until further analysis. Mice were all sacrificed at the same time point.

\subsection{Metabolic characterization}

Animals were weighed and body composition was assessed by MR scanning (EchoMRI ${ }^{\mathrm{TM}}$, USA). At the age of 14 and 15 weeks an oral glucose tolerance test (OGTT) and an insulin tolerance test (ITT) were performed, respectively. Both tests were performed after a 6-hour fast and blood glucose measurements were done from the tail vein. For the OGTT a bolus of glucose (25\% glucose/saline solution, $2 \mathrm{~g}$ glucose/kglean body mass) was delivered by oral gavage, and tail vein blood glucose was measured just before and at 20, 40, 60, 90 and 120- minutes after the glucose bolus. The ITT was performed following 8 days of recovery after the OGTT. Insulin $(100 \mathrm{U} / \mathrm{ml})$ was diluted in gelofusine (B. Braun, Denmark) for a working solution of $0.333 \mathrm{U} / \mathrm{ml}$, and administered by intraperitoneal injection $(0.75 \mathrm{U} / \mathrm{kg}$ lean body mass $)$. Blood glucose in the tail blood was measured before and at 15, 30, 45, 60, 90 and 120-minutes after the injection. The mice were conscious, and placed in their cages during both tests. A week before collecting tissues, $200 \mu \mathrm{l}$ of blood was drawn from the mandible into EDTA coated tubes, left to clot at room temperature for 30 -minutes and spun down at $2000 \times g$ at $4{ }^{\circ} \mathrm{C}$ for 10 -minutes. Supernatants were transferred to clean tubes and stored at $-80^{\circ} \mathrm{C}$

\subsection{Triacylglycerol measurement of liver tissue}


Published in final edited form as: Molecular and Cellular Endocrinology 412 (2015) 65-72 doi:10.1016/i.mce.2015.05.028

${ }^{1} \mathrm{H}$-high resolution magic-angle spinning-nuclear magnetic

resonance spectroscopy. Five to fifteen milligrams of intact liver tissue was transferred into a $4 \mathrm{~mm}$ zirconia HR-MAS rotor with a volume of $15 \mu \mathrm{l}$. Additionally, $5 \mu \mathrm{l}$ of a $100 \mu \mathrm{M}$ solution of trisodium phos- phate was added as reference. The NMR spectra were recorded using a $600 \mathrm{MHz}$ Bruker Avance III NMR spectrometer (Bruker, Rheinstetten, Germany). All measurements were conducted under MAS at a frequency of $9 \mathrm{kHz}$ and a temperature of $25{ }^{\circ} \mathrm{C}$. The water signal was suppressed by presaturation ( $1 \mathrm{~mW}$ power for $3 \mathrm{~s}$ ). NMR spectra were excited by a $\pi / 2$ pulse of $4 \mu$ s duration. To allow for complete relaxation, the delay time between successive scans was $30 \mathrm{~s}$. The amount of TAG was determined by comparing the inte- grals of the trisodium phosphate signal with the glycerol backbone signal at $4.29 \mathrm{ppm}$ which is exclusively found in TAG. All spectra were corrected for baseline and phase distortions using Spinworks (University of Manitoba). Deconvolution of the signals was per- formed using an in-house written SciPy script. The peaks were fitted to a Voigt-Profile applying a constrained least-squares approach based on the L-BGFGS optimization algorithm (Zhu et al., 1997). The TAG content is expressed per gram liver tissue.

\subsection{Protein extraction, western blot analyses and immunoprecipitation}

Approximately $10 \mathrm{mg}$ liver tissue was lysed in modified RIPA buffer as previously described (Schuster et al., 2014). Protein con- centration was determined using Pierce BCA protein assay (Thermo Scientific) and equal amounts of protein were separated by SDS- PAGE and transferred using a semi-dry transfer apparatus to nitrocellulose membranes. Next, membranes were blocked in 5\% non- fat dry milk in TBS buffer containing $0.1 \%$ Tween 20. Applied antibodies are listed in Supplementary Table S1. Detection of pro- teins was carried out using Luminata Classico Western HRP Substrate (Merck Millipore) or Amersham ECL Prime Western Blotting De- tection Reagent (GE Healthcare). Gapdh immunoblotting was performed as a loading control. For immunoprecipitation livers were incubated with anti-acetyl lysine antibody conjugated to $\mu$ MACS Protein A Micro Beads (Miltenyi Biotec) overnight and eluted with $1 \times$ SDS sample buffer. After 5 min heating, samples were analyzed by Western blotting with an anti-Srebp1 antibody (H-160, Santa Cruz Biotechnology).

\subsection{Total RNA extraction and realtime $q P C R$}

Total RNA of liver tissue was extracted by TRIzol ${ }^{\circledR}$ Reagent (Life Technologies) according to manufacturer's protocol. One micro- gram of total RNA was transcribed into cDNA by M-MLV Reverse Transcriptase (Invitrogen). Quantitative PCR analyses were performed using the qPCR Master Mix Plus Low ROX (Eurogentec) or Absolute qPCR SYBR Green Low ROX Mix (Thermo Scientific) and the Applied Biosystems 7500 Real Time PCR System. Primer se- quences are summarized in Supplementary Table S2.

\subsection{NAD measurement}

NAD was measured by EnzyChrom ${ }^{\mathrm{TM}} \mathrm{NAD}^{+} / \mathrm{NADH}$ Assay Kit (E2ND-100, Biotrend) according to manufacturer's protocol or reversed-phase HPLC using the Chromaster Purospher STAR RP- 18 endcapped $3 \mu \mathrm{m}$ Hibar RT 150-3 HPLC column (Merck). Ten milligrams of frozen liver tissue was sonicated in $100 \mu 11 \mathrm{M}$ per- chloric acid. After a 10-minute incubation period on ice samples were centrifuged and the supernatant was neutralized with $3 \mathrm{M}$ potas- sium carbonate. After repeated centrifugation samples were loaded onto the column as previously described (Schuster et al., 2014).

\subsection{Nampt enzyme activity}

For determination of Nampt activity $10 \mathrm{mg}$ of liver tissue was lysed in $100 \mu \mathrm{l}$ Nampt enzyme assay buffer (0.1 M sodium phos- phate, $\mathrm{pH} 7.4)$ and protein concentration was determined using BCA protein assay. Thirty micrograms of protein was added to the re- action buffer and incubated at $37{ }^{\circ} \mathrm{C}$ for $1 \mathrm{~h}$. Afterwards the assay using radiolabeled ${ }^{14} \mathrm{C}$-nicotinamide was performed as previously described (Garten et al., 2010).

\subsection{Serum Nampt measurement}

Nampt in mouse serum was measured by the Nampt (Visfatin/ PBEF) (mouse/rat) Dual ELISA Kit (AdipoGen Inc., Seoul, South Korea), according to manufacturer's instructions.

\subsection{Statistical analysis}

Statistical analyses were performed with the open source lan- guage R in version 3.0.2 for triacylglycerol measurements or with GraphPad Prism ${ }^{\circledR}$ software (5.03) for all other statistical compari- sons. Significance levels were calculated by unpaired, two-tailed 
t-tests or by repeated two-way ANOVA with subsequent Bonferroni posttest where appropriate. For TAG measurement of liver tissue a twotailed Kruskal-Wallis test was conducted to determine sig- nificant differences between $\mathrm{Ctl}$ and HFD groups, because data were not normally distributed. Data represent mean \pm SEM. $p<0.05$ was considered significant. $* \mathrm{p}<0.05 ; * * \mathrm{p}<0.01 ; * * * \mathrm{p}<0.001$ compared to $\mathrm{Ctl}$ mice.

\section{Results}

3.1. Mice fed a high-fat diet have impaired glucose tolerance and increased hepatic lipid content while markers for fibrosis and inflammation are not altered

To determine how a HFD affects hepatic NAD metabolism, a cohort of mice were put on a HFD (60\% of total energy from fat) or Ctl diet for 11 weeks at 6 weeks of age. After 11 weeks on HFD, mice were significantly heavier $(39.0 \pm 4.2 \mathrm{~g}$ vs. $29.9 \pm 2.5 \mathrm{~g})$ (Fig. 1A) and had significantly increased amounts of body fat $(13.3 \pm 1.1 \mathrm{vs}$.

$3.3 \pm 0.6 \mathrm{~g}$ ) (Fig. 1B) compared to Ctl mice. Lean body mass was significantly higher in Ctl vs. HFD mice $(25.5 \pm 0.5$ vs. $23.0 \pm 0.3 \mathrm{~g})$

(Fig. 1C). HFD mice showed a significantly impaired oral glucose
A)
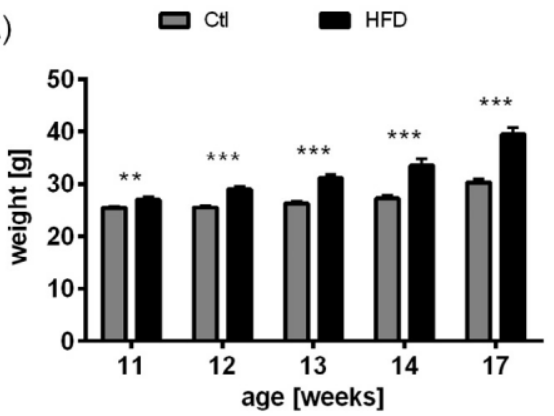

D)

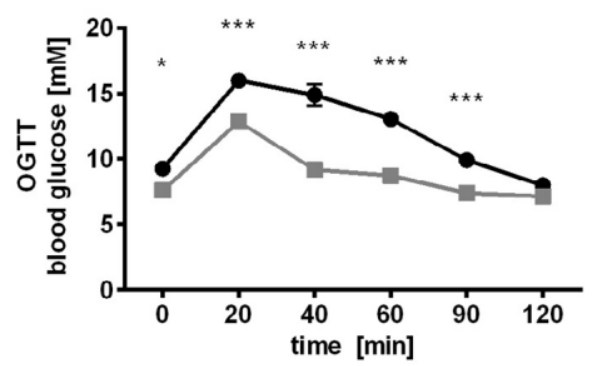

G)

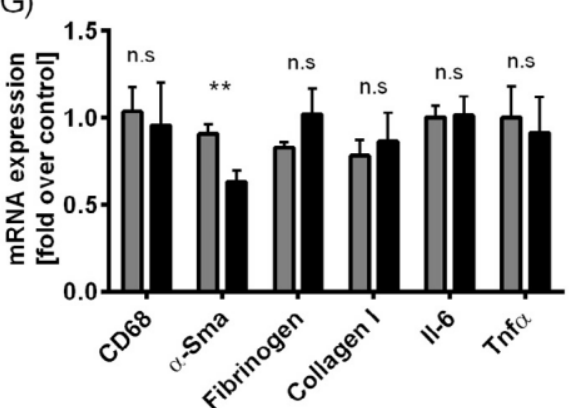

B)

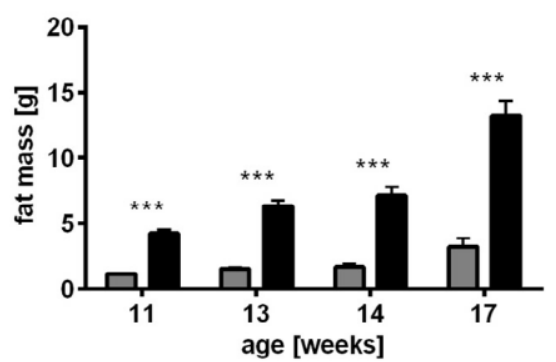

E)

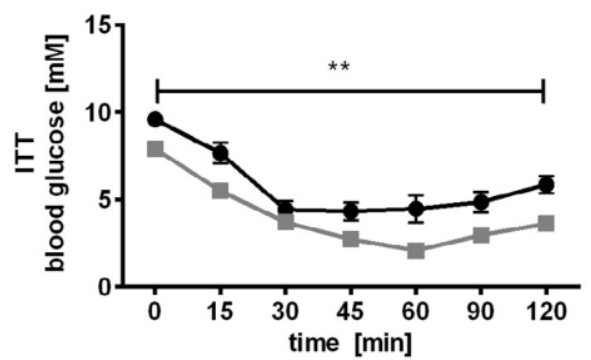

C)

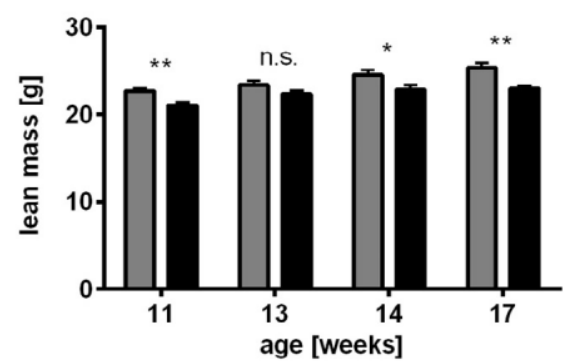

F)

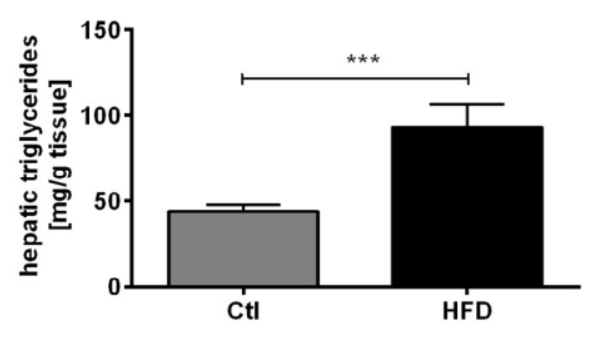

Fig. 1. Mice fed a high fat diet have impaired glucose tolerance and increased hepatic lipid content while markers for fibrosis and inflammation are not altered. Mice fed a high fat diet (HFD) for 11 weeks gained weight (A) and fat body mass (B) while lean body mass (C) was decreased compared to controls (Ctl). The HFD mice showed an impaired oral glucose tolerance (D) and insulin tolerance (E) compared to Ctl. Livers of HFD mice showed higher levels of triglycerides (F) but no indication for macrophage infiltration as measured by CD68 expression. $\alpha$-Sma was significantly down regulated in livers of mice fed a HFD while fibrinogen and collagen I expression as markers for fibrosis stayed stable. mRNA expressions of markers for inflammation (TNF $\alpha$ and, Il-6) were unchanged in both groups (G). Data are shown as means \pm SEM. n.s., not significant,

$* \mathrm{p}<0.05, * * \mathrm{p}<0.01$ and $* * * \mathrm{p}<0.001$ compared to Ctl mice. 
tolerance $(\mathrm{p}<0.001)$. Significant differences in fasting blood glucose as well as at 20, 40, 60 and 90 min were found between the Ctl and HFD groups (Fig. 1D). In the insulin tolerance test a main effect of diet $(\mathrm{p}=$ $0.0018)$ and a borderline significant interaction effect were observed $(\mathrm{p}=$ 0.055 ; observed power, $\mathrm{P}=0.78$ ) (Fig. 1E). Hepatic TAG content was 2.1fold increased compared to Ctl mice $(93.2 \pm 12.6$ vs. $44.1 \pm 3.8 \mathrm{mg} / \mathrm{g}$ tissue) (Fig. 1F). To further evaluate the state of NAFLD we measured a marker for macrophage infiltration, $C D 68$, which was not different between both groups. $\alpha$-smooth muscle actin $(\alpha-$ Sma $)$ was decreased in HFD mice while other markers for fibro- sis (fibrinogen, collagen $I$ ) were not different. Interleukin-6 (Il-6) and tumor necrosis factor alpha (Tnfo) as markers for inflammation were not changed in HFD mice (Fig. 1G). mRNA expression of the cytokines chemokine $(C-X-C$ motif) ligand 16 (Cxcl16), monocyte chemotactic protein 1 (Mcp-I) and transforming growth factor $\beta(\operatorname{Tg} \beta \beta)$ were not different in the livers of mice fed a HFD compared to Ctl. mRNA ex- pression of the co receptor of the T cell receptor $C D 8$ and the fatty acid translocase $C D 36$ were not changed in the livers of the HFD mice (Supplementary Fig. S2A). Carnitine palmitoyltransferase (Cpt)-I $\alpha$ and- II are two mitochondrial enzymes which are important for $\beta$ oxidation of long-chain fatty acids. Both stayed stable in mice fed a HFD (Supplementary Fig. S2B). Furthermore, we detected less en- doplasmic reticulum stress in livers of HFD mice indicated by lower levels of phosphorylated eIF $2 \alpha$ compared to Ctl mice (Supplementary Fig. S2C).

\subsection{High-fat diet up regulates hepatic Nampt mRNA expression, protein levels, activity, and NAD levels}

Nampt levels have been shown to be either up or down regu- lated in different studies on insulin resistance and glucose intolerance (Friebe et al., 2011; Goktas et al., 2013; Olszanecka-Glinianowicz et al., 2014). Interestingly, both mRNA and protein levels of Nampt were increased in HFD mice compared with Ctl mice by 1.9-fold and 1.7-fold, respectively (Fig. 2A,B). This was in line with a significant 1.5 -fold increase in Nampt enzyme activity $(41.4 \pm 4.2$ vs. $65.8 \pm 5.8 \mathrm{cpm} / \mu \mathrm{g}$ protein $\times \mathrm{h})$ in the HFD fed mice (Fig. 2C). Because Nampt is the key regulator of the mammalian NAD salvage pathway we measured hepatic NAD levels and found that NAD was signifi- cantly increased by 1.6-fold in the HFD mice compared to Ctl mice ( $4.2 \pm 0.3$ vs. $6.7 \pm 0.5 \mathrm{nmol} / \mathrm{mg}$ protein) (Fig. 2D). Since hepato- cytes are able to secrete Nampt (Garten et al., 2010), we measured Nampt in mouse serum samples from the mice. However, no dif- ferences between HFD and Ctl group were observed $(19.8 \pm 4.9$ vs. $14.6 \pm 1.3 \mathrm{ng} / \mathrm{ml}$ ) (Fig. 2E).

\subsection{Acetylation of Nfkb and p53 is decreased while Sirt1 protein is increased in HFD mice}

Since NAD is a required substrate for deacetylases and since total hepatic acetylation of the HFD mice was down regulated (Supplementary Fig. $\mathrm{S} 3 \mathrm{~A}$ ), we measured the acetylation state of $\mathrm{Nfkb}$ (ac-Nfkb) and p53 (acp53), which are involved in the regulation of inflammation and apoptosis, respectively (Yeung et al., 2004). Total NfkB protein was up regulated in livers of HFD mice compared to $\mathrm{Ctl}$ mice while the acetylation of $\mathrm{NfkB}$ stayed stable resulting in 2.1- fold less acetylated NfאB (Fig. 3A). We found that total and phosphorylated p53 protein was not changed (Supplementary Fig. S2D), while acetylated p53 was significantly reduced by 1.8- fold (Fig. $3 \mathrm{~A}$ ), implicating that p53 was less active in murine livers of HFD mice compared to $\mathrm{Ctl}$ mice. In support of this finding, Bax, a downstream target of p53, was decreased by 1.6-fold (Fig. 3A). As NAD levels were up regulated resulting in lower levels of acety- lated p53 and $\mathrm{NfkB}$, we further investigated protein and mRNA levels
A)

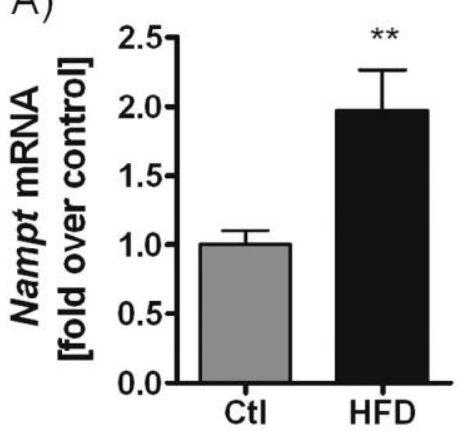

C)

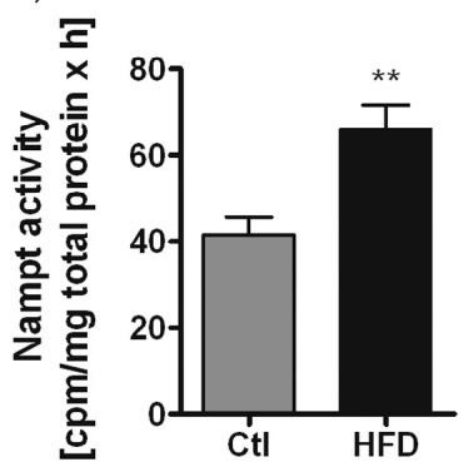

B)

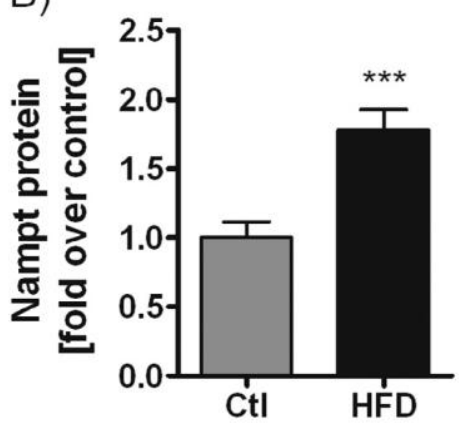

E)

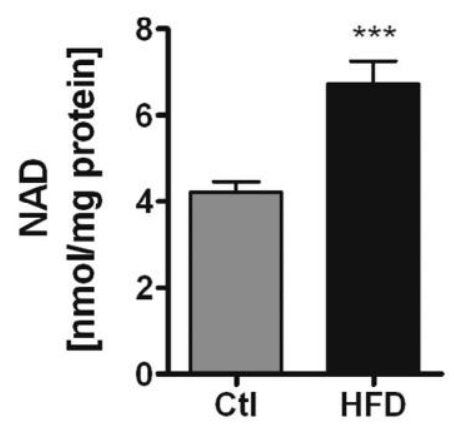

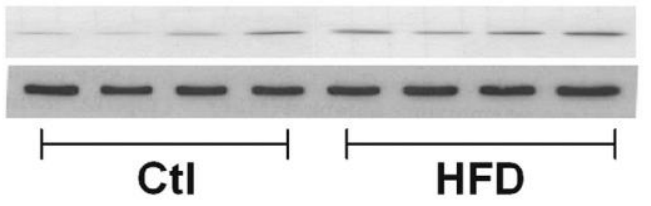

Nampt Gapdh

Fig. 2. HFD up regulates hepatic Nampt expression and activity as well as NAD levels. After a HFD for 11 weeks murine livers showed an increased Nampt mRNA (A) and protein amount (B) measured by qPCR or western blot analyses, respectively, compared to Ctl mice. Nampt activity (C) and intracellular NAD levels (D) measured by HPLC were enhanced. Serum Nampt levels were unchanged between both groups (E). Data are shown as means $\pm \operatorname{SEM}(\mathrm{n}=12)$. One representative Western Blot is shown. **p<0.01 and $* * * \mathrm{p}<0.001$ compared to Ctl mice.

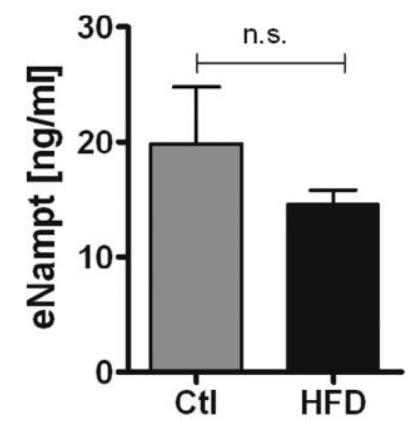



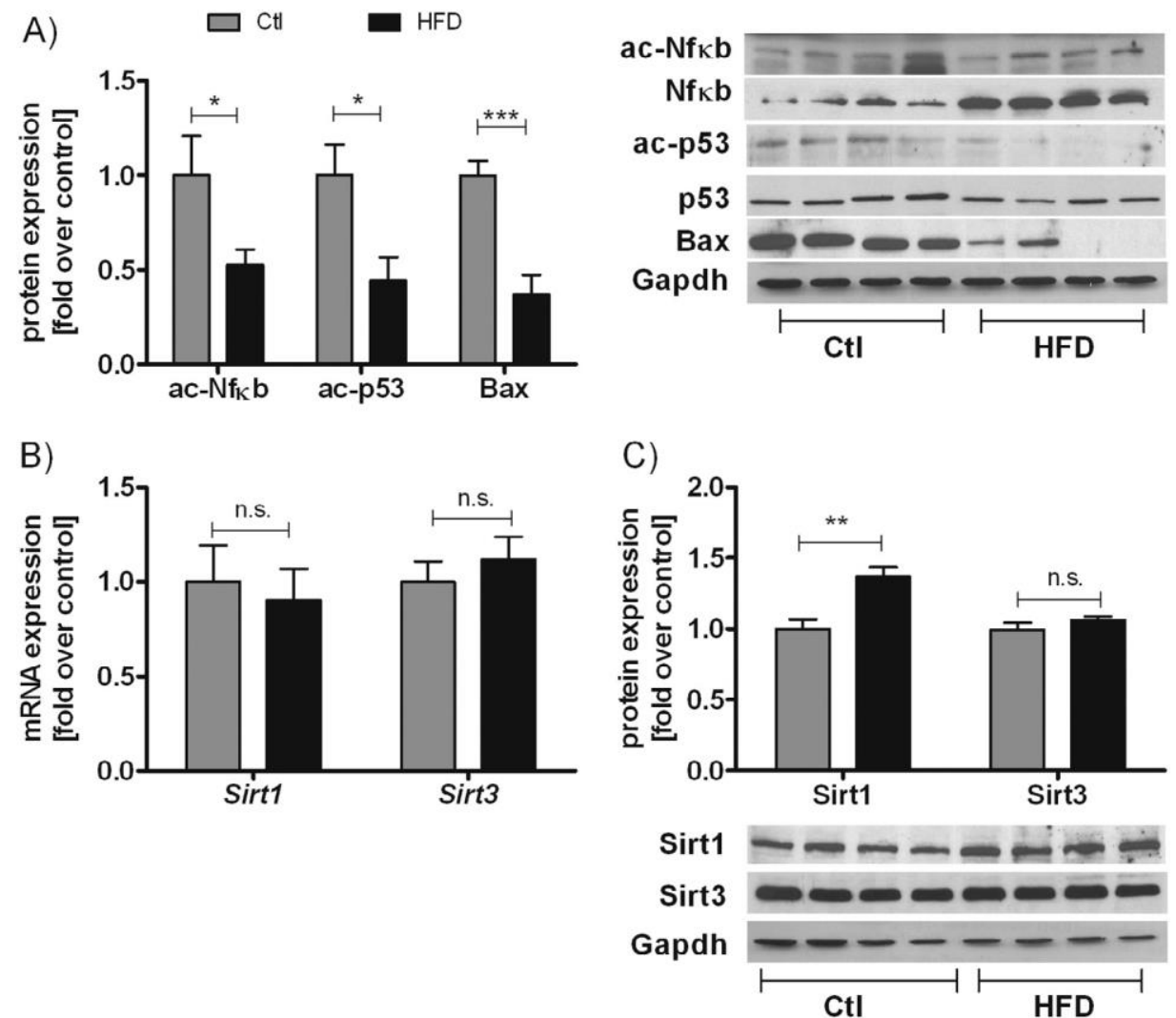

Fig. 3. Acetylation state of Nfib and p53 are down regulated in HFD mice which is associated with increased Sirt1 protein expression. The acetylation status of Nfik (ac-Nfikb), acetylated p53 (ac-p53) and total Bax protein were decreased in livers of mice fed a HFD compared to Ctl mice as shown by densitometric analysis of Western blots (A). Sirt1 and Sirt3 mRNA (B) and protein levels (C) in liver tissues were measured by qPCR and Western blot analyses, respectively. Hepatic Sirt1 protein was up regu- lated in mice fed a HFD for 11 weeks compared to Ctl mice. Data are shown as means \pm SEM $(n=12)$. One representative Western blot is shown. Acetylated protein is normalized to the corresponding total protein. Gapdh is used as loading control n.s., not significant, $* \mathrm{p}<0.05, * * \mathrm{p}<0.01$ and $* * * \mathrm{p}<0.001$ compared to Ctl mice.

of Sirt1 and Sirt3 which are proteins known to be regulated during NAFLD development (Hirschey et al., 2010; Purushotham et al., 2009). No differences in Sirt1 and Sirt3 mRNA levels were detected between groups (Fig. 3B). However, Sirt1 protein was significantly in- creased by 1.4-fold in the HFD group compared to $\mathrm{Ctl}$ while Sirt3 protein amount stayed stable (Fig. $3 C)$.

\subsection{Expression of sirtuin downstream targets is altered in} mice fed a $H F D$

The expression of further sirtuin targets that are associated with the pathogenesis of NAFLD was analyzed. Mitochondrial uncoupling protein 2 (Ucp 2 ) was significantly reduced by 1.4 -fold in HFD mice compared to $\mathrm{Ctl}$ mice while peroxisome proliferator-activated recep- tor gamma coactivator $1 \alpha(P g c-1 \alpha)$ and mitochondrial superoxide dismutase $(\mathrm{MnSOD})$, markers of the mitochondrial biogenesis, were unchanged (Fig. 4A). Sirt3 deacetylates MnSOD at K122 resulting in increased MnSOD activity (Tao et al., 2010). Using an antibody tar- geting acetylated MnSOD K122 (kindly provided by Prof. David Gius, North Westwestern University) we measured the acetylation state of MnSOD and found no differences on total protein and acetyla- tion level (Supplementary Fig. S3B). Sirt1 is a transcriptional regulator of sterol regulatory element-binding protein 1 (Srebp-1c) (Defour et al., 2012), a key regulator of de novo lipogenesis in the liver. Srebp-1c was enhanced by 1.9-fold while its downstream targets fatty acid synthase (Fas), stearoyl-CoA-desaturase $1(S c d 1)$ and acetyl-coA-
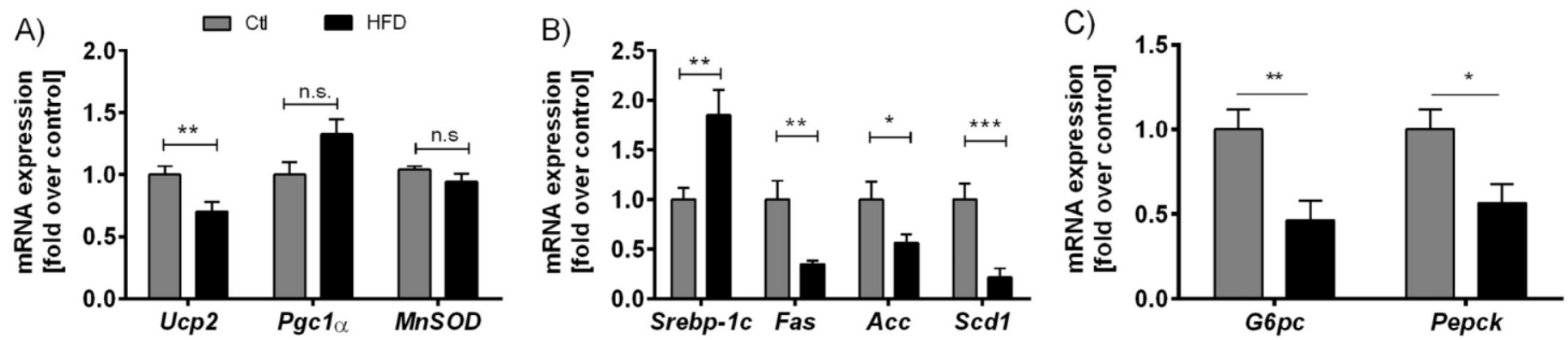

Fig. 4. Sirt 1 downstream targets were altered in mice fed a HFD. HFD mice showed a reduced expression of Ucp 2 while Pgc1 $\alpha$ and MnSOD were unchanged (A) as ana- lyzed by qPCR Srebp-1c mRNA expression was up regulated while Fas, Acc and Scd1 mRNA expression was declined compared to Ctl mice (B). G6pc and Pepck were both decreased in mice fed a HFD (C). n.s., not significant, $* \mathrm{p}<0.05, * * \mathrm{p}<0.01$ and $* * * \mathrm{p}<0.001$ compared to Ctl mice. 
carboxylase (Acc) were significantly declined (Fig. 4B). Acetylation of Srebp-1 was not significantly different in both groups (Supplementary Fig. S3C). Glucose-6-phosphatase (G6pc) and phosphoenolpyruvate carboxykinase (Pepck), key enzymes of gluconeogenesis, were both downregulated by 1.8 -fold and 2.2- fold, respectively, in the livers of the HFD mice compared to control chow (Fig. $4 C)$.

\section{Discussion}

The impact of hepatic lipid accumulation on NAD metabolism in the liver is incompletely known. To this end we evaluated the effect of a HFD intervention in mice on the hepatic NAD salvage pathway. The HFD mouse model employed in this study represents the human phenotype of hepatosteatosis with increased hepatic TAG accumulation, but without any indications of macrophage infiltra- tion or fibrosis. Our data are also in accordance with data from other studies using HFD mice

(Takahashi et al., 2012). Mice on HFD had impaired glucose tolerance, higher fasting blood glucose levels as well as a borderline significant reduction in insulin tolerance com- pared to mice on normal chow. Collectively, this indicates that HFD-

fed mice had decreased peripheral insulin sensitivity.

Interestingly, we found an up regulation in the capacity for NAD salvage in the livers of the HFD-fed mice, indicated by up regulated expression and activity of the NAD salvage enzyme Nampt and in- creased NAD levels. The enhanced NAD salvage we observed could be a prerequisite for the up regulation of mitochondrial metabo- lism as an adaptive mechanism in response to chronic fat overload as described in other studies (Koliaki and Roden, 2013), since an increase in $\beta$-oxidation and tricarboxylic acid cycleflux would require increased levels of NAD. In general, it has been shown that mice fed a HFD exhibit decreased hepatic Nampt levels, while an up reg- ulation of Nampt ameliorated the negative impact of HFD in the liver and augmented glucose tolerance (Tao et al., 2011; Yoshino et al., 2011). In contrast, and in accordance with our study, another study showed an up regulation of Nampt mRNA in a rat model of NAFLD (Chang et al., 2014). Reasons for the discrepant results of animal studies could be the composition and duration of the HFD as well as mouse strain-dependent differences (Heiker et al., 2014). Con- flicting data about the role of hepatic Nampt in humans have been reported. In obese women hepatic NAMPT mRNA levels in the liver were shown to be higher compared to lean women and were even furtherincreased in obese women with NAFLD, which is in line with our data (Auguet et al., 2013). A different human study found higher NAMPTmRNA expression level in fibrotic livers (Kuklaetal., 2010). Opposite, it has also been reported that patients with NAFLD showed lower levels of NAMPT mRNA expression in the liver(Dahl et al.,2010). The reason for the discrepancies between these studies is unknown but it could be due to variations in the progression of NAFLD.

As a result of increased NAD levels an enhanced Sirt1 deacetylase activity in the livers of the HFD-fed mice was detected as shown by less acetylation of Nf-kb on lysine 310 (Yeung et al., 2004) and p53 on lysine 382 (Vaziri et al., 2001). By deacetylating p53 at lysine 382 Sirt1 is able to repress the activity of p53, a master regulator of apoptosis and cell cycle progression (Vaziri et al., 2001). In liver biopsies from subjects with NAFLD Sirt1 was shown to be de- creased leading to increased acetylation of p53 compared to Ctl patients (Castro et al., 2013). The lower activity of p53 in the present study was confirmed by the observed down regulation of the p53 downstream target Bax in HFD murine livers (Rozan and El-Deiry, 2007). Nf- $\kappa b$ is a critical factor in the innate immunity response and is directly affected by Sirt 1 which deacetylates the ReIA/p65 com- ponent of Nf-kb leading to a down regulation of its activity and degradation (Yeung et al., 2004). A global over expression of Sirt1 in mice subjected to HFD has been shown to improve hepatic inflammation by decreasing activity of Nf-kb (Pfluger et al., 2008).
The increased Sirt1 activity in HFD-fed mice was confirmed by detecting a down regulation of Ucp2. This mitochondrial inner membrane protein negatively regulates reactive oxygen species (ROS) production and has been demonstrated by others to be up regulated in NAFLD (Kohjima et al., 2007). Sirt1 is known to regulate Ucp2 by binding to its promoter resulting in down regulation of Ucp 2 mRNA expression (Bordone et al., 2006). Our data indicate that a higher ac- tivity of Sirt1 led to a down regulation of $U c p 2$ in HFD mouse livers suggesting that oxidative stress was not present in our murine model at the analysed time point. The absence of oxidative stress was sup- ported by the fact that MnSod, a key ROS scavenging enzyme (Tao et al., 2010), was not different on mRNA level as well as on total and acetylated protein level in our murine model.

Srebp-1c can be inhibited by Sirt1 and is a key regulator of de novo lipogenesis, which plays a crucial role in the pathogenesis of NAFLD Srebp-1c is mainly activated by saturated fatty acids and elevated insulin levels (Aragno et al., 2009; Haas et al., 2012). In line with these studies, we found higher Srebp-1c mRNA expression in livers of HFD-fed mice. Despite increased TAG accumulation in the liver of HFD-fed mice it might however be reasonably assumed that Srebp-1c was less active due to deacetylation by Sirt1 (Ponugoti et al., 2010), emphasized by lower mRNA levels of its downstream targets Fas, Acc and Scd1. However, in our study acetylation of Srebp-1 was not significantly altered in mice fed a HFD compared to Ctl. Tran- scriptional activity of Srebp-1c is also regulated by phosphorylation due to activation of AMPK which suppresses Srebp-1c cleavage and nuclear translocation in insulin resistant LDL receptor deficient mice and therefore represses de novo lipogenesis (Li et al., 2011). Interestingly we could also find a decreased expression of the two key enzymes of gluconeogenesis G6pc and Pepck which might be due to higher activity of Sirt1 leading to an increased phosphorylation (Wang et al., 2011) and decreased acetylation of FoxO1 (Motta et al., 2004) and thus inhibiting the transcription of G6pc and Pepck

Besides elevated Sirt 1 activity, we found increased hepatic Sirt1 protein expression in HFD-fed mice. There are several contradic- tory studies on Sirt1 expression in the livers of NAFLD animal models. Rats on a HFD for 3 months were reported to develop hepatic ste- atosis accompanied by decreased Sirt1 protein levels (Deng et al., 2007), while in other studies Sirt1 over expression was found to be protective against the negative impact of a HFD (Pfluger et al., 2008; Purushotham et al., 2009). In contrast, it has been shown that liver- specific Sirt1 knockout mice stored less fat in white adipose tissue and liver under HFD conditions (Chen et al., 2008). In addition, liver- specific Sirt1 knockout mice were more glucose tolerant and had lower levels of blood glucose and insulin than wild type controls (Chen et al., 2008). Taken together, the association between Sirt1 protein and activity levels, hepatic lipid accumulation and result- ing metabolic disturbances is not yet clarified and may be associated with disease progression and inflammatory conditions.

In summary, we detected an up regulation of the NAD salvage pathway and concomitantly increased Sirt 1 deacetylase activity in the livers of mice fed a HFD diet for 11 weeks. One could suppose that up regulation of Nampt activity and NAD levels is an early com- pensatory mechanism to protect the liver against the negative consequences of lipid accumulation. The negative impact of an 11- week HFD was being counteracted via higher Nampt and Sirt1 activities. It is possible that a more prolonged HFD exposure would lead to impaired regulation of hepatic Nampt and NAD levels, thus contributing to the development of steatohepatitis.

\section{Funding}

This publication is supported by the German Competence Network Obesity, Federal Ministry of Education and Research (grant number: 01GI1330) and LIFE - Leipzig Research Center for Civili- zation Diseases, Universität Leipzig. LIFE is funded by means of the 
European Union, by the European Regional Development Fund (ERDF) (grant number: 4-7531.70/5/4) and by means of the Free State of Saxony within the framework of the excellence initiative. We want to thank the Deutsche Forschungsgemeinschaft SFB 1052, "Obesity Mechanisms" (B06) for support. Support for this project was also provided by the Novo Nordisk Foundation Center for Basic Meta- bolic Research. This work was supported by a research grant from the Danish Diabetes Academy supported by the Novo Nordisk Foun- dation. The Novo Nordisk Foundation Center for Basic Metabolic Research is an independent Research Center at the University of Co- penhagen partially funded by an unrestricted donation from the Novo Nordisk Foundation (www.metabol.ku.dk). Jonas T. Treebak was supported by the Novo Nordisk Foundation (Excellence Project Award) (grant number: NNF14OC0009315), the Danish Council for Inde- pendent Research | Medical Sciences (grant number: 4004-00235) and the European Foundation for the Study of Diabetes (EFSD/ Lilly Research Fellowship Programme).

\section{Acknowledgement}

The authors wish to acknowledge the skilled technical exper- tise of Anja Barnikol-Oettler, Steve Risis and Thomas S. Nielsen. We thank the Research Academy Leipzig and the Integrated Research Training Group "Obesity Mechanisms"(CRC 1052) for support and we thank Prof. David Gius (Northwestern University Feinberg School of Medicine, Chicago, IL, USA) for generously providing the acetylation-specific MnSOD antibody.

MP, SS, MD, BJ, TG, SGV, JT, WK and AG conceived experiments, MP, PL, AM and SR carried out experiments, MP, MD, PL, AM, JT and AG analyzed data. All authors were involved in writing the paper and had final approval of the submitted and published versions.

\section{Appendix: Supplementary material}

Supplementary data to this article can be found online at doi:10.1016/j.mce.2015.05.028.

\section{References}

Aragno, M., Tomasinelli, C.E., Vercellinatto, I., Catalano, M.G., Collino, M., Fantozzi, R., et al., 2009. SREBP-1c in nonalcoholic fatty liver disease induced by Western- type highfat diet plus fructose in rats. Free Radic. Biol. Med. 47, 1067-1074 doi:10.1016/j.freeradbiomed.2009.07.016.

Auguet, T., Terra, X., Porras, J.A., Orellana-Gavaldà, J.M., Martinez, S., Aguilar, C., et al., 2013. Plasma visfatin levels and gene expression in morbidly obese women with associated fatty liver disease. Clin. Biochem. 46, 202-208. doi:10.1016/ j.clinbiochem.2012.11.006.

Bordone, L., Motta, M.C., Picard, F., Robinson, A., Jhala, U.S., Apfeld, J., et al., 2006. Sirt1 regulates insulin secretion by repressing UCP2 in pancreatic beta cells. PLoS Biol. 4, e31. doi:10.1371/journal.pbio.0040031.

Castro, R.E., Ferreira, D.M.S., Afonso, M.B., Borralho, P.M., Machado, M., V, Cortez-Pinto, H., et al., 2013. miR-34a/SIRT1/p53 is suppressed by ursodeoxycholic acid in the rat liver and activated by disease severity in human non-alcoholic fatty liver disease. J. Hepatol. 58, 119-125. doi: 10.1016/j.jhep.2012.08.008.

Chang, W.-C., Jia, H., Aw, W., Saito, K., Hasegawa, S., Kato, H., 2014. Beneficial effects of soluble dietary Jerusalem artichoke (Helianthus tuberosus) in the prevention of the onset of type 2 diabetes and non-alcoholic fatty liver disease in high- fructose diet-fed rats. of type 2 diabetes and non-alcoholic fatty liver disease in
Br. J. Nutr. 112, 709-717. doi:10.1017/S0007114514001421

Chen, D., Bruno, J., Easlon, E., Lin, S.-J., Cheng, H.-L., Alt, F.W., et al., 2008. Tissue-specific regulation of SIRT1 by calorie restriction. Genes Dev. 22, 1753-1757. doi:10.1101/ gad. 1650608 .

Dahl, T.B., Haukeland, J.W., Yndestad, A., Ranheim, T., Gladhaug, I.P., Damås, J.K., et al., 2010. Intracellular nicotinamide phosphoribosyltransferase protects against hepatocyte apoptosis and is down-regulated in nonalcoholic fatty liver disease. J. Clin. Endocrinol. Metab. 95, 3039-3047. doi:10.1210/jc.2009-2148.

Defour, A., Dessalle, K., Castro Perez, A., Poyot, T., Castells, J., Gallot, Y.S., et al., 2012. Sirtuin 1 regulates SREBP-1c expression in a LXR-dependent manner in skeletal muscle. PLoS ONE 7, e43490. doi:10.1371/journal.pone.0043490.

Deng, X.-Q., Chen, L.-L., Li, N.-X., 2007. The expression of SIRT1 in nonalcoholic fatty liver disease induced by high-fat diet in rats. Liver Int. 27, 708-715. doi:10.1111/ j.14783231.2007.01497.x.

Fernandez-Marcos, P.J., Jeninga, E.H., Canto, C., Harach, T., de Boer, V.C.J., Andreux, P., et al., 2012. Muscle or liver-specific Sirt3 deficiency induces hyperacetylation of mitochondrial proteins without affecting global metabolic homeostasis. Sci. Rep. 2, mitochondrial proteins without
425. doi: $10.1038 /$ srep00425.

Friebe, D., Neef, M., Kratzsch, J., Erbs, S., Dittrich, K., Garten, A., et al., 2011. Leucocytes are a major source of circulating nicotinamide phosphoribosyltransferase (NAMPT)/pre-B cell colony (PBEF)/visfatin linking obesity and inflammation in humans. Diabetologia 54, 1200-1211. doi:10.1007/s00125-010-2042-z.

Garten, A., Petzold, S., Barnikol-Oettler, A., Körner, A., Thasler, W.E., Kratzsch, J., et al., 2010. Nicotinamide phosphoribosyltransferase (NAMPT/PBEF/visfatin) is constitutively released from human hepatocytes. Biochem. Biophys. Res. Commun. 391, constitutively released from human hepatocy

Goktas, Z., Owens, S., Boylan, M., Syn, D., Shen, C.-L., Reed, D.B., et al., 2013. Associations between tissue visfatin/nicotinamide, phosphoribosyltransferase (Nampt), retinol binding protein-4, and vaspin concentrations and insulin resistance in morbidly obese subjects. Mediators Inflamm. 2013, 861496. doi: $10.1155 / 2013 / 861496$

Haas, J.T., Miao, J., Chanda, D., Wang, Y., Zhao, E., Haas, M.E., et al., 2012. Hepatic insulin signaling is required for obesity-dependent expression of SREBP-1c mRNA but not for feeding-dependent expression. Cell Metab. 15, 873-884. doi:10.1016/ j.cmet.2012.05.002.

Heiker, J.T., Kunath, A., Kosacka, J., Flehmig, G., Knigge, A., Kern, M., et al., 2014 Identification of genetic loci associated with different responses to high-fat diet-induced obesity in C57BL/6N and C57BL/6J substrains. Physiol. Genomics 46, 377-384. doi:10.1152/physiolgenomics.00014.2014.

Hirschey, M.D., Shimazu, T., Goetzman, E., Jing, E., Schwer, B., Lombard, D.B., et al., 2010 SIRT3 regulates mitochondrial fatty-acid oxidation by reversible enzyme deacetylation. Nature 464, 121-125. doi:10.1038/nature08778.

Kohjima, M., Enjoji, M., Higuchi, N., Kato, M., Kotoh, K., Yoshimoto, T., et al., 2007. Reevaluation of fatty acid metabolism-related gene expression in nonalcoholic fatty liver disease. Int. J. Mol. Med. 20, 351-358

Koliaki, C., Roden, M., 2013. Hepatic energy metabolism in human diabetes mellitus, obesity and non-alcoholic fatty liver disease. Mol. Cell. Endocrinol. 379, 35-42. doi:10.1016/j.mce.2013.06.002.

Kukla, M., Ciupiń ska-Kajor, M., Kajor, M., Wyleż oł, M., Ż wirska-Korczala, K., Hartleb, M., et al., 2010. Liver visfatin expression in morbidly obese patients with nonalcoholic fatty liver disease undergoing bariatric surgery. Pol. J. Pathol. 61, 147 153

Li, Y., Xu, S., Mihaylova, M.M., Zheng, B., Hou, X., Jiang, B., et al., 2011. AMPK phosphorylates and inhibits SREBP activity to attenuate hepatic steatosis and atherosclerosis in diet-induced insulin-resistant mice. Cell Metab. 13, 376-388. doi:10.1016/j.cmet.2011.03.009.

Motta, M.C., Divecha, N., Lemieux, M., Kamel, C., Chen, D., Gu, W., et al., 2004. Mammalian SIRT1 represses forkhead transcription factors. Cell 116, 551- 563.

Olszanecka-Glinianowicz, M., Owczarek, A., Boż entowicz-Wikarek, M., Brzozowska, A., Mossakowska, M., Zdrojewski, T., et al., 2014. Relationship between circulating visfatin/NAMPT, nutritional status and insulin resistance in an elderly from the PolSenior substudy. Metabolism 63, 1409-1418. doi:10.1016/j.metabol 2014.07.013.

Pfluger, P.T., Herranz, D., Velasco-Miguel, S., Serrano, M., Tschöp, M.H., 2008. Sirt1 protects against high-fat diet-induced metabolic damage. Proc. Natl. Acad. Sci. U.S.A. protects against high-fat diet-induced metabolic d

Ponugoti, B., Kim, D.-H., Xiao, Z., Smith, Z., Miao, J., Zang, M., et al., 2010. SIRT1 deacetylates and inhibits SREBP-1C activity in regulation of hepatic lipid metabolism. J. Biol. Chem. 285, 33959-33970. doi:10.1074/jbc.M110.122978.

Purushotham, A., Schug, T.T., Xu, Q., Surapureddi, S., Guo, X., Li, X., 2009. Hepatocyte- specific deletion of SIRT1 alters fatty acid metabolism and results in hepatic steatosis and inflammation. Cell Metab. 9, 327-338. doi:10.1016/j.cmet .2009 .02 .006

Revollo, J.R., Grimm, A.A., Imai, S., 2007a. The regulation of nicotinamide adenine dinucleotide biosynthesis by Nampt/PBEF/visfatin in mammals. Curr. Opin. Gastroenterol. 23, 164-170. doi:10.1097/MOG.0b013e32801b3c8f.

Revollo, J.R., Korner, A., Mills, K.F., Satoh, A., Wang, T., Garten, A., et al., 2007b. Nampt/PBEF/Visfatin regulates insulin secretion in beta cells as a systemic NAD biosynthetic enzyme. Cell Metab. 6, 363-375. doi:10.1016/j.cmet.2007.09 .003 .

Rozan, L.M., El-Deiry, W.S., 2007. p53 downstream target genes and tumor suppression: a classical view in evolution. Cell Death Differ. 14, 3-9. doi:10.1038/ sj.cdd. 4402058 .

Samal, B., Sun, Y., Stearns, G., Xie, C., Suggs, S., McNiece, I., 1994. Cloning and characterization of the cDNA encoding a novel human pre-B-cell colonyenhancing factor. Mol. Cell. Biol. 14, 1431-1437.

Samuel, V.T., Liu, Z.-X., Qu, X., Elder, B.D., Bilz, S., Befroy, D., et al., 2004. Mechanism of hepatic insulin resistance in non-alcoholic fatty liver disease. J. Biol. Chem. 279, 32345-32353. doi: 10.1074/jbc.M313478200.

Schuster, S., Penke, M., Gorski, T., Petzold-Quinque, S., Damm, G., Gebhardt, R., et al., 2014 Resveratrol differentially regulates NAMPT and SIRT1 in hepatocarcinoma cells and primary human hepatocytes. PLoS ONE 9, e91045. doi:10.1371/ journal.pone.0091045.

Takahashi, Y., Soejima, Y., Fukusato, T., 2012. Animal models of nonalcoholic fatty liver disease/nonalcoholic steatohepatitis. World J. Gastroenterol. 18, 2300-2308. doi:10.3748/wjg.v18.i19.2300.

Tanaka, M., Nozaki, M., Fukuhara, A., Segawa, K., Aoki, N., Matsuda, M., et al., 2007. Visfatin is released from 3T3-L1 adipocytes via a non-classical pathway. Biochem. Biophys. Res. Commun. 359, 194-201. doi:10.1016/j.bbrc.2007.05.096.

Tao, R., Coleman, M.C., Pennington, J.D., Ozden, O., Park, S.-H., Jiang, H., et al., 2010. Sirt3-mediated deacetylation of evolutionarily conserved lysine 122 regulates MnSOD activity in response to stress. Mol. Cell 40, 893-904. doi:10.1016/ j.molcel.2010.12.013

Tao, R., Wei, D., Gao, H., Liu, Y., Depinho, R.A., Dong, X.C., 2011. Hepatic FoxOs regulate lipid metabolism via modulation of expression of the nicotinamide phosphoribosyltransferase gene. J. Biol. Chem. 286, 14681-14690. doi:10.1074/ jbc.M110.201061.

Tarantino, G., Finelli, C., 2013. What about non-alcoholic fatty liver disease as a new criterion to define metabolic syndrome? World J. Gastroenterol. 19, 3375-3384. doi:10.3748/wjg.v19.i22.3375.

Turner, N., Kowalski, G.M., Leslie, S.J., Risis, S., Yang, C., Lee-Young, R.S., et al., 2013. Distinct patterns of tissue-specific lipid accumulation during the induction of insulin resistance in mice by high-fat feeding. Diabetologia 56, 1638-1648 doi:10.1007/s00125-013-2913-1.

Vaziri, H., Dessain, S.K., Ng Eaton, E., Imai, S.I., Frye, R.A., Pandita, T.K., et al., 2001. hSIR2 (SIRT1) functions as an NAD-dependent p53 deacetylase. Cell 107, 149-159.

Wang, R.-H., Kim, H.-S., Xiao, C., Xu, X., Gavrilova, O., Deng, C.-X., 2011. Hepatic Sirt deficiency in mice impairs mTorc2/Akt signaling and results in hyperglycemia, oxidative damage, and insulin resistance. J. Clin. Invest. 121, 4477-4490. doi:10.1172/JCI46243.

Yeung, F., Hoberg, J.E., Ramsey, C.S., Keller, M.D., Jones, D.R., Frye, R.A., et al., 2004 Modulation of NF-kappaB-dependent transcription and cell survival by the SIRT1 deacetylase. EMBO J. 23, 2369-2380. doi:10.1038/sj.emboj.7600244.

Yoshino, J., Mills, K.F., Yoon, M.J., Imai, S., 2011. Nicotinamide mononucleotide, a key NAD+ intermediate, treats the pathophysiology of diet- and age-induced diabetes in mice. Cell Metab. 14, 528-536. doi:10.1016/j.cmet.2011.08.014

Zhu, C., Byrd, R.H., Lu, P., Nocedal, J., 1997. Algorithm 778: L-BFGS-B: Fortran subroutines for large-scale bound-constrained optimization. ACM Trans. Math. Softw. 23 , 550-560. doi: $10.1145 / 279232.279236$. 